\title{
A Simple Thermoelectric Droplet Generator
}

\author{
Andrew Work ${ }^{1}$, Mario Vargas², Yongsheng Lian ${ }^{3}$ \\ $\left({ }^{1}\right.$ Ohio Aerospace Institute, corresponding author, email address: \\ andrew.h.work@nasa.gov)
}

( ${ }^{2}$ NASA Glenn Research Center)

$\left({ }^{3}\right.$ University of Louisville)

Pre-print, published edition available here:

\begin{abstract}
A new design for a droplet generator capable of producing single droplets is presented. The design relies on thermoelectric heating to vaporize water at the interface between a droplet and a blunt syringe tip. While other designs require careful tuning to produce drops of varying size, this technique enables the simple creation of droplets of any size within a range. The design is of simple construction and can be completed with off-the-shelf components, and relies on resistive heating to vaporize water at or near the droplet-nozzle interface and release the droplets. We demonstrated that the design can be used to produce droplets as small as $110 \mu \mathrm{m}$ or as large as $2 \mathrm{~mm}$. Drop size is limited by the geometry of the nozzle since water must wet the tip of the nozzle and hang under gravity. Our experiments showed that released droplets have relatively small disturbances introduced by the release mechanism when compared to competing techniques. These disturbances were intermittently observed as the voltage, pulse width, and drop size were changed, and optimal settings were determined for the smallest drop sizes produced.
\end{abstract}

\section{Introduction}

The production of single droplets of water is critical to the study of droplet impact, which is important to a wide variety of fields; one such field is aircraft icing [1], others include inkjet printing, spray coating processes, and fire suppression. Current generators can produce single droplets of water, but rely on relatively complicated mechanisms to produce singular droplets of a particular size. When single droplets are produced, they are highly disturbed droplets possessing large oscillations of fluid motion within the droplet as inertial forces compete with surface tension forces. In experiments where a small distance is required between the nozzle and the test area, these oscillations result in a non-spherical droplet - e.g. a droplet splash may occur with an oblong drop when a spherical drop is required to compare to other results. In most existing droplet generators used, water is forced through a nozzle and violently separated from the nozzle [2-10]. Breaking contact with the nozzle requires that local surface tension forces holding the droplet be defeated; in general, this results in a violent separation process and highly disturbed droplets. These designs force water through a nozzle into a jet, and rely on Rayleigh instability to form droplets. This physical requirement creates a complicated relationship between input parameters and the size of the created droplet. Available single droplet generator designs include piezoelectric designs [2, 5, 7-13], thermal bubble designs [6, 14, 15], pneumatic designs [16, 17], ultrasonic designs [3, 4], vibrating orifice designs (to produce a monodisperse stream of droplets) [18], and filament designs [19]. All of these designs mechanically break droplet contact with the nozzle.

Piezoelectric designs rely on a piezoelectric actuator to pulse water through a nozzle, where Rayleigh instability brings the ejected water into a spherical shape [10]. In these designs, water is contained in a pressured reservoir with a nozzle exit [9]. A piezoelectric actuator pulses the water, forcing water through the nozzle (and back into the reservoir). Early designs were complicated. Newer designs are much more simplistic, but it is still difficult to use these designs to produce clean droplets of any desired size since the drop is still formed through the same complex mechanisms where momentum, surface tension, and Rayleigh instability are competing. The thermal bubble method is another approach to produce droplets in printers [14]. This method utilizes a resistive heater inside a 
reservoir behind a nozzle and pulses a heater to vaporize and force the ink out by pressurizing the reservoir, similarly to piezoelectric designs.

The difficulty with both piezoelectric and thermal bubble designs in producing droplets of arbitrary size is that the exact relationship between the electrical pulse and the size of the droplets produced by the generator is unknown. For example, pulsing either the heater or the piezoelectric actuator twice as long will almost certainly not result in a droplet twice as large. Piezoelectric disks possess linear displacement, but the amount of fluid expelled through the nozzle may not be linearly related to the displacement of the piezo element due to dynamic effects such as compliance in the structure, or back-feeding flow into the supply. With both thermal bubble and piezoelectric designs, the flow through the nozzle competes against surface tension, viscous forces, and the momentum of the fluid. Water that exits the nozzle must be pinched off through surface tension instabilities or be pulled back into the nozzle. With either design, the production of a droplet of a particular size requires careful experimentation. Even when a suitably sized droplet is produced, the droplet may be of poor quality making it unsuitable for use in the study of droplet physics (e.g. non-spherical, off-center droplets).

In the study of droplet splashing and breakup, high quality droplets are required to make meaningful comparisons between data sets. Droplets produced by current droplet generators are inadequate to study the relevant phenomena in many cases, and the effort required to repeatedly produce droplets of a particular size or even in a spherical geometry is non-trivial [20]. The difficulty with which this need is met with current designs is documented in the literature. Dong et. al. performed a series of experiments to investigate how to produce quality droplets under varying conditions [21]. They show how secondary droplets can form from liquid threads between droplets, how droplets can recombine, how droplets may oscillate, and how the generator inputs must be tweaked to optimize droplet production.

In order to get the quality of drop required for our studies in aircraft icing, we designed a novel droplet generator capable of producing single drops of water that is the archetype of a class of thermal droplet generators. Our design is similar to the thermal bubble design, except the heat is not used to create a pressure to mechanically separate the droplet - it is instead used to vaporize the water at the interface to break surface tension directly. The design of this droplet generator is presented here along with experiments that were conducted.

\section{Generator Design}

Instead of breaking the surface tension mechanically, the design provided herein relies on the thermal vaporization of the water at the interface between the droplet and the nozzle to break the surface tension. In this design, shown in Figure 1, stainless steel nozzles were heated directly using resistive heating. 


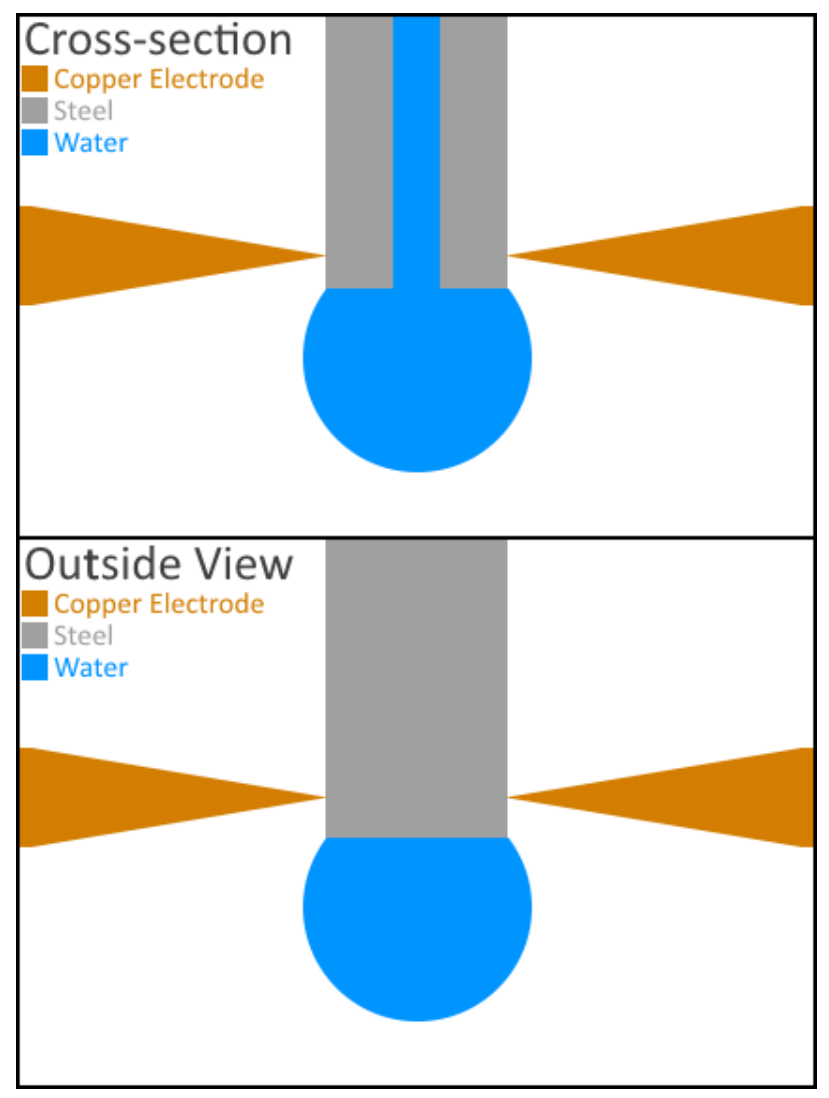

Figure 1. Design diagram, directly heated stainless-steel nozzle. Not to scale.

The design utilized commercially available syringe tips of three different sizes to produce a variety of droplet sizes. Two blunt, stainless steel syringe tips from Nordson EFD were used, a Lavendar 30g tip (310 $\mu \mathrm{m}$ O.D., 30g is 30 gage), and a Yellow 32g tip (240 $\mu$ m O.D.). One tip was used from World Precision Instruments Inc, a blunt, stainless steel Nanofil $36 \mathrm{~g}$ nozzle (110 $\mu \mathrm{m}$ O.D.). Smaller nozzles enabled the creation of smaller droplets.

Copper electrodes were fashioned from copper plate by cutting out strips and filing the ends to a point. A small flat was added to the end to increase the contact area. The use of copper and stainless steel in the design was convenient since the copper would melt to form better contact around the syringe tip. As shown in Figure 2, the copper electrodes melted to form good contact around the stainless steel tubing. This limits the use of materials selected since the electrodes should have a lower melting point than the heating element material. Three sets of electrodes were made. The first two sets of electrodes were similar and used on the 30g and 32g nozzles. The third set of electrodes was used to test the $36 \mathrm{~g}$ nozzle, and to run two tests on the 32g nozzle. The first two sets were only filed while the third set was polished using up to 4000 grit silicon carbide paper. 


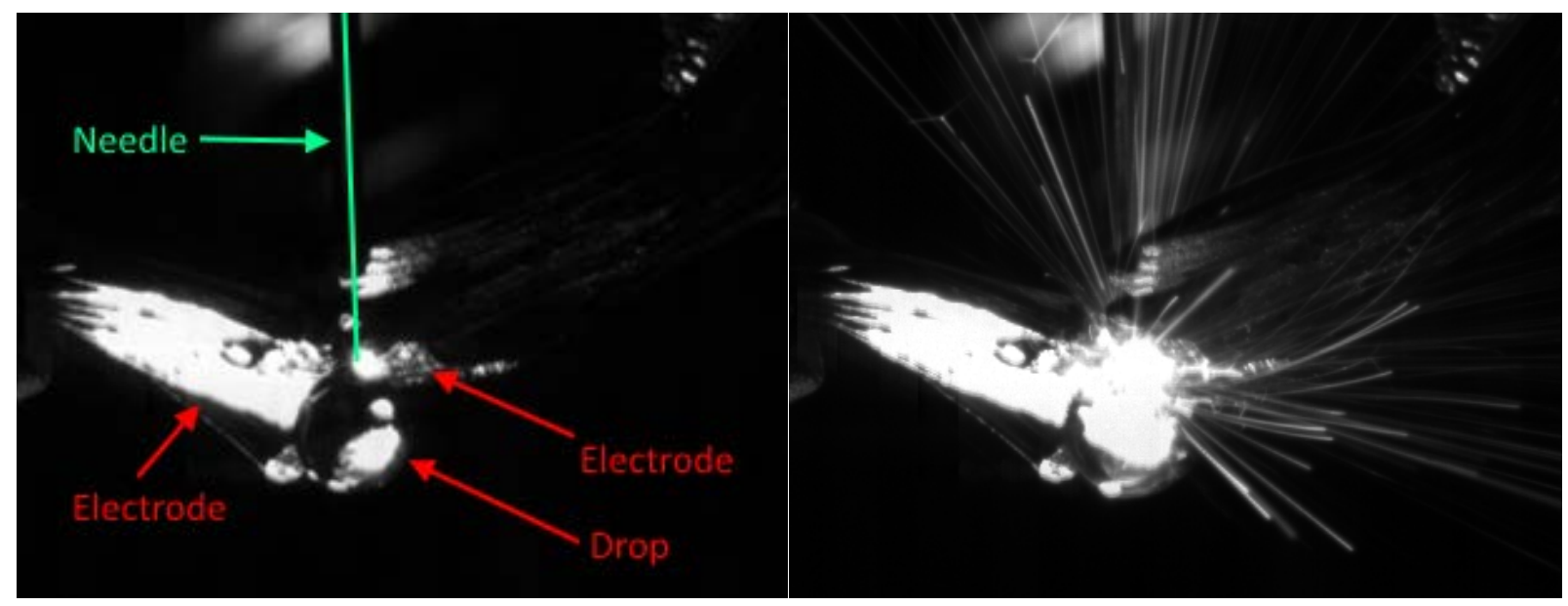

Figure 2. The first droplet release, using 32g needle and rough electrodes. Excessive molten copper can be seen on right as electrodes wear in, to be followed by a violent droplet release.

In the design, when electricity is pulsed across the tip, resistive heating will follow the path of least resistance - the shortest path between the electrodes. This will result in a band of metal inside the needle tip being heated as shown in the top left of Figure 3. The heat from this initial band will dissipate and vaporize water inside the needle, creating a bubble just upstream of the droplet. This bubble will force water out of the needle, disturbing the droplet.

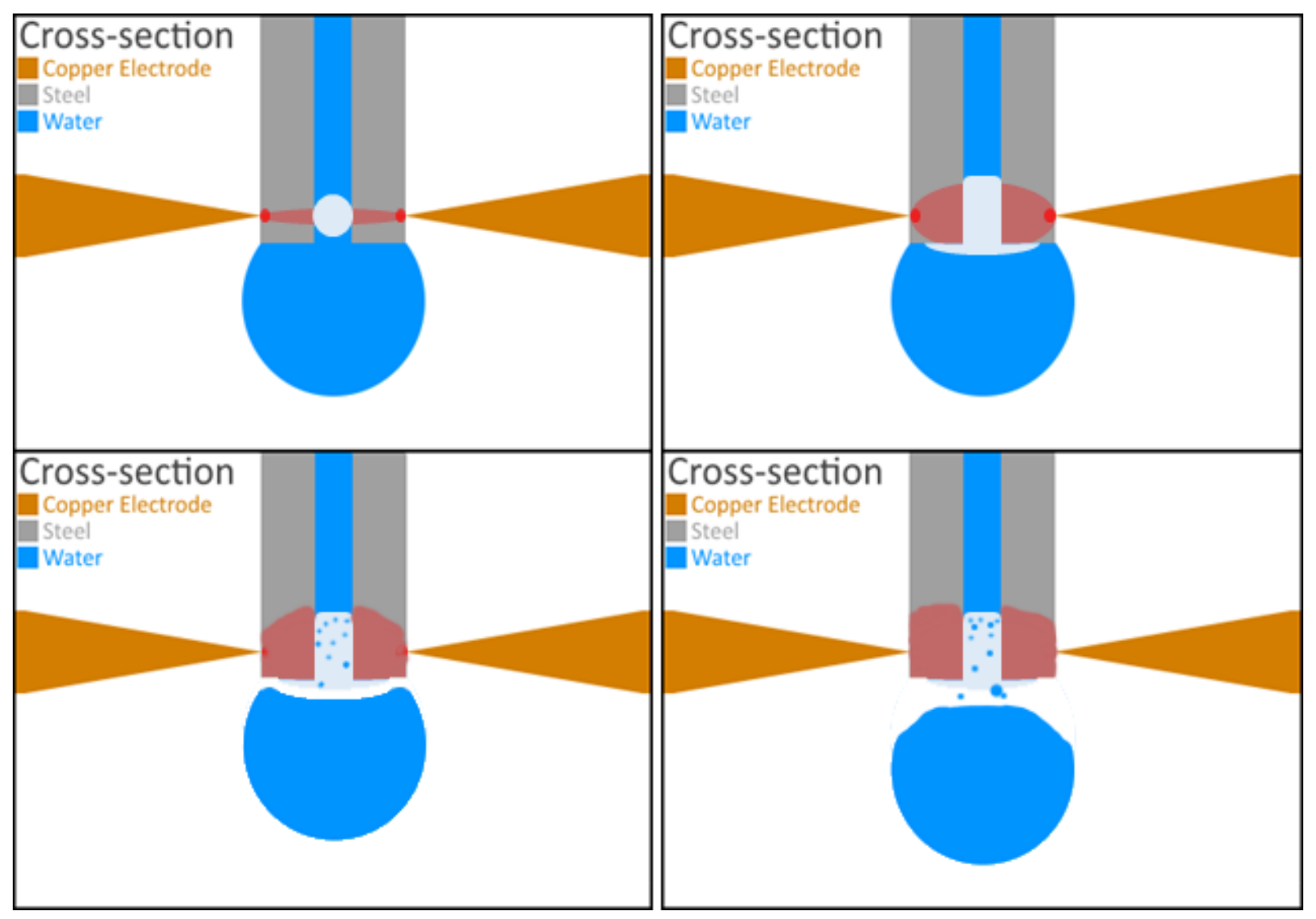

Figure 3. Depiction of heat and vaporization in droplet separation. 


\section{Apparatus Design}

The electrical equipment used to power the droplet generator is shown in Figure 4. A capacitor was charged using a bench top power supply (Agilent 6032A) and allowed to discharge across the syringe tip over short time periods on the millisecond time scale. Two power transistors (FUJI Electric EVG31-050) were used in parallel to control the discharge. The power transistors were controlled with a Field Effect Transistor (FET, model IRF541), which was in turn controlled by an arbitrary waveform generator (Wavetek 395) that generated a 5V pulse. The FET was powered by another desktop power supply set to 2V (BK Precision 1746). It was found that the FET was necessary since the Wavetek did not generate enough current to consistently control the power transistors.
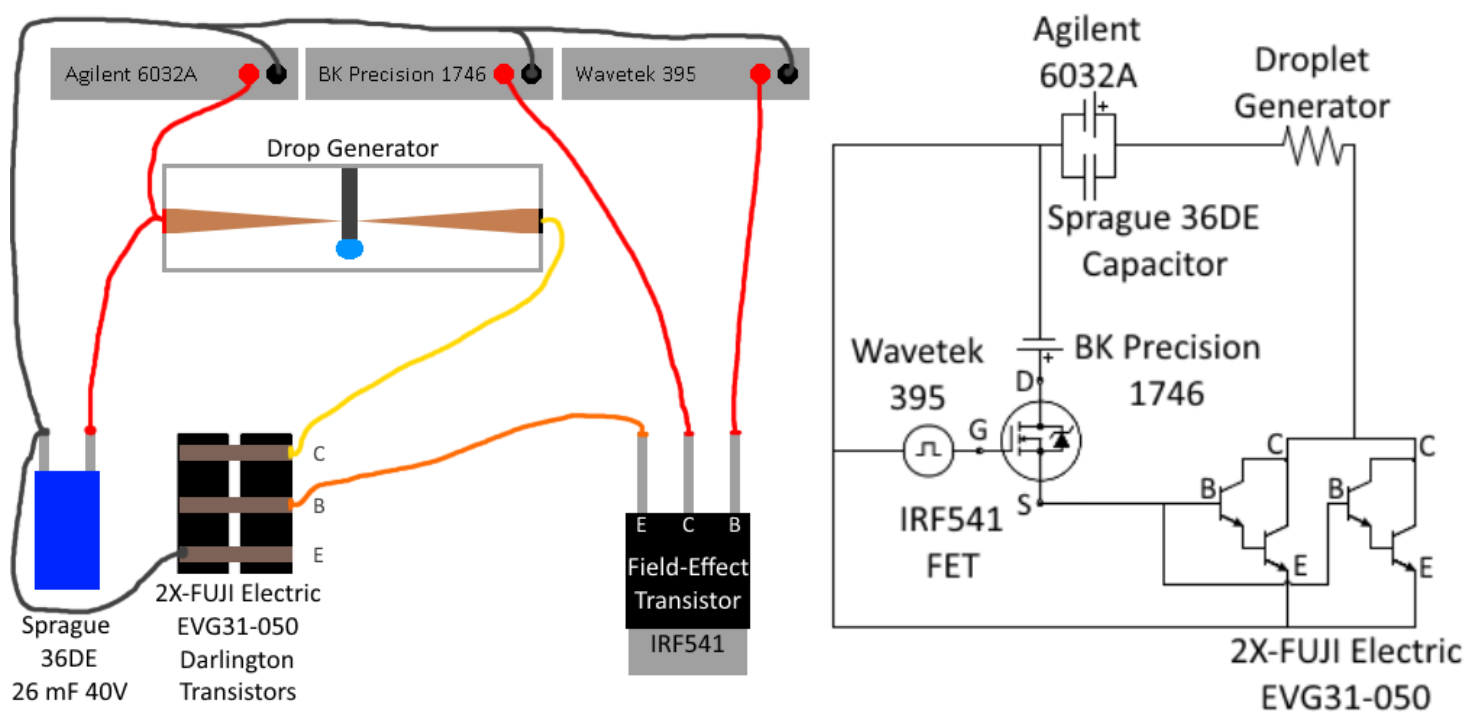

Figure 4. Left: Apparatus electrical diagram, not to scale. Right: Circuit diagram.

Voltages from 2 to $40 \mathrm{~V}$ were used to heat the nozzles used, with pulse widths between $50 \mu \mathrm{s}$ and $10 \mathrm{~ms}$. It was found that much higher amounts of power were needed for larger tips since they possessed more thermal mass and lower electrical resistance across the tip, increasing power dissipation elsewhere in the circuit. The 36g nozzle required much less power and was run using less than 3V across the capacitor. To record droplet ejection, a Phantom V10 camera was used with an Infinity Photo-Optical Company lens, which can be seen at the left side of Figure 5. The droplets were backlit using an Arrilux 200 lamp. 


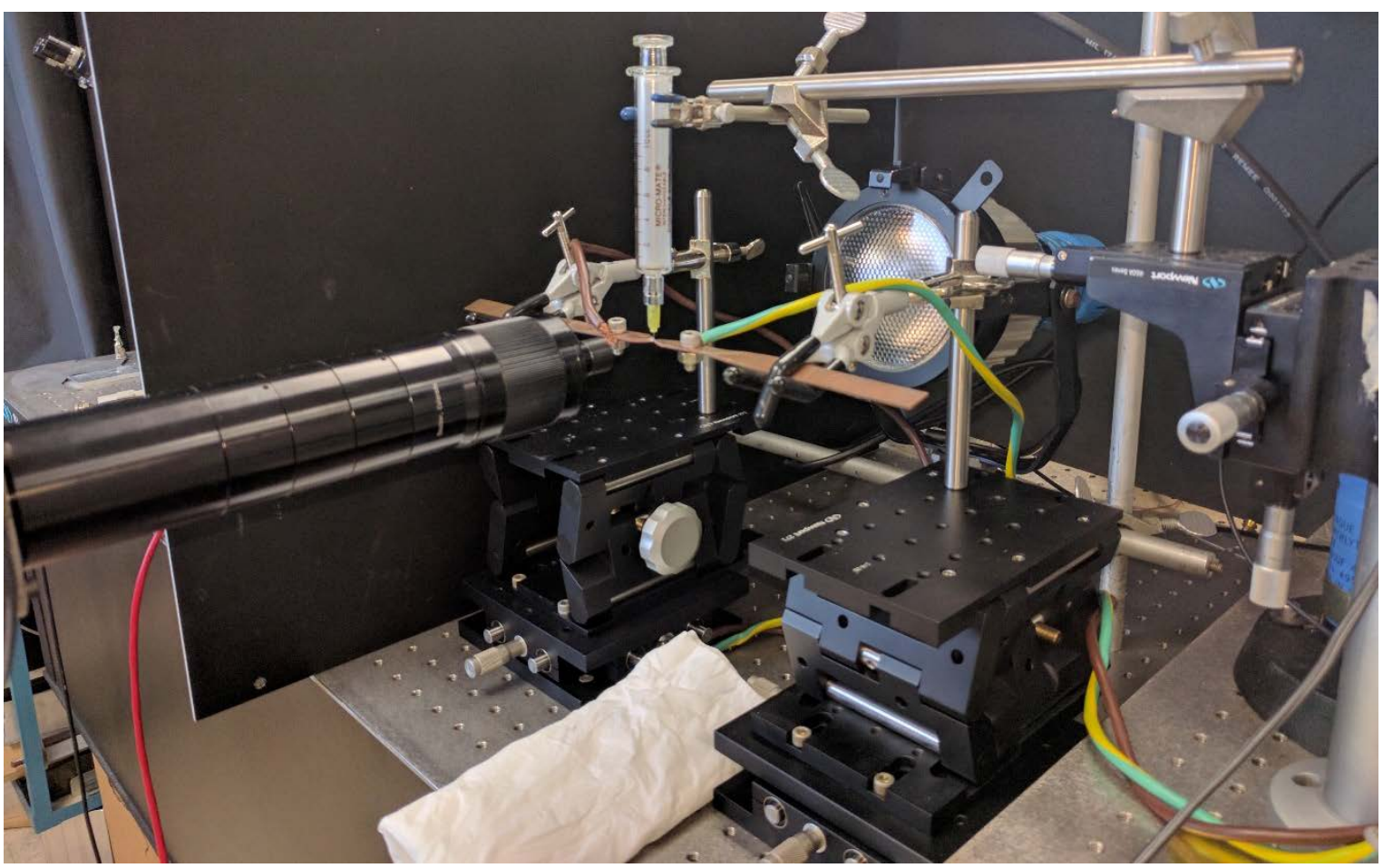

Figure 5. Experimental setup (lens \& camera at left).

Events were manually triggered and recorded in Phantom Camera Control (PCC) 2.1 at frame rates of either 4 or $24 \mathrm{kHz}$, with exposure times ranging from 20-700 $\mu$ s. The pulse width was visually verified at the capacitor and at the function generator using an Agilent MSO7104A oscilloscope. Newport lab jacks and 2-axis translation stages were used to control the position of each of the electrodes and a 3-axis stage was used to control the position of the nozzle. Droplets were produced by manually pressing the syringe. The system was not mechanically isolated from the building even though it was mounted to a vibration isolation table (the table supports were depressurized).

\section{Results \& Discussion}

In the experiments with the droplet generator, we varied the voltage, pulse width, electrode position, and drop size to produce different results. Priority was given to smaller droplets due to the difficulty in producing these droplets; it was found that larger droplets could be produced reliably with little difficulty using this design and so these sizes are under-represented in the results presented here. In general, electrodes were placed as close to the tip as possible while preventing drops from contacting the electrodes. Droplet contact on the electrodes was a primary cause for 'stick' cases where droplets were not ejected or recorded. The electrode position was not recorded for the first two nozzles used but it was observable that further placement from the tip resulted in poorer droplets. Moving the electrodes up resulted in more water being vaporized upstream of the droplet, and a more mechanical separation of the droplet. It also resulted in larger post-separation spray, and if high enough, caused a column of water to shoot through droplets suspended from the end of the nozzle. The polished electrodes used throughout the experiments with the $36 \mathrm{~g}$ nozzle allowed for the contact position of the electrodes to be determined from recordings for most cases. Of the 32 recordings with the $36 \mathrm{~g}$ nozzle, the position could not be determined in cases 9 and 16 .

Three methods were used to determine the distance of the electrode on the left side of the recording with respect to the nozzle tip. The first method (Dist 1) took the average of the top and bottom coordinates of the electrode where they intersected the left boundary of the nozzle, and subtracted the vertical coordinate of the tip. The second method (Dist 2) used the intersection point of two lines, each tracing the top or bottom edge of the electrode, and subtracted the vertical coordinate for this point from the vertical coordinate of the tip. The third method (Dist 3) used the lower intersection point from the first method and subtracted the bottom. This allowed for distance calculations for cases 11-15 and 17, in which the top of the electrode was not recorded. The position of the right electrode was 
kept close to the left electrode, and only the left electrode position was calculated. The points used for each method are shown in Figure 6. An example of applying these methods to an actual case, case 30, are shown in Figure 7.

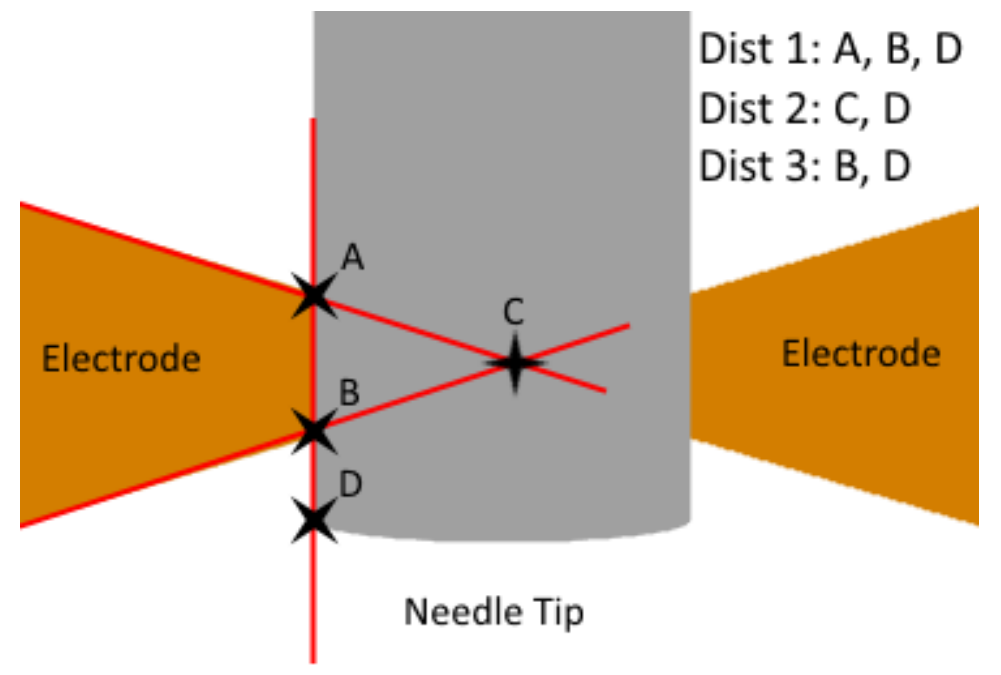

Figure 6. Points used in each distance calculation.

Nearly all droplets released produced small secondary droplets as water in the syringe tip was vaporized. The expulsion of vapor frequently was observed to push the drops, and often caused them to visibly shake. This is seen in Figure 7 where the images 13 and 23 capture the spray of droplets. In this case, the largest secondary droplets were caught by the primary drop. The effects were intermittent and difficult to predict. Lowering the electrodes to their lowest point possible helped prevent this spray. The white spot seen on the drops was due to the light source.

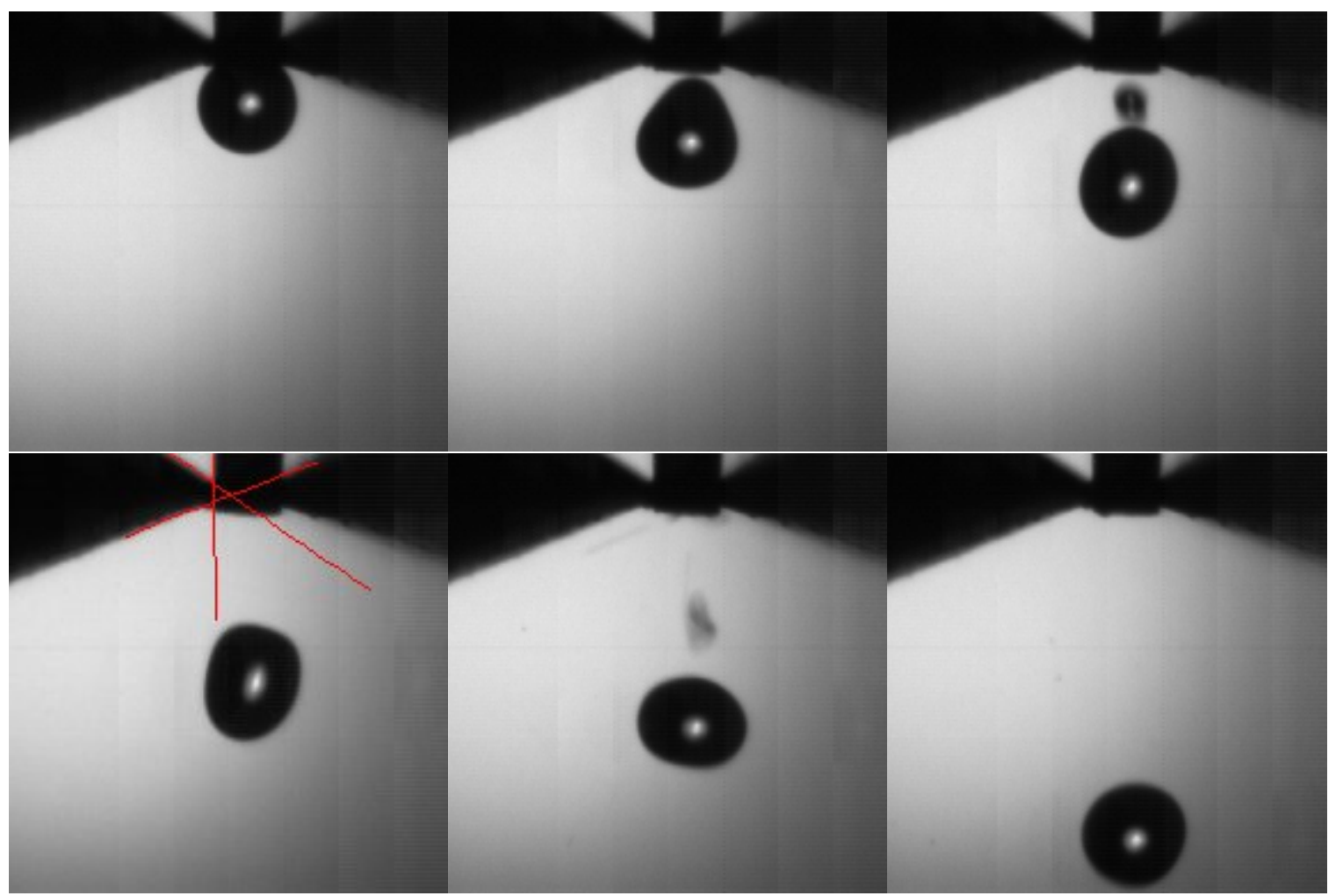

Figure 7. Case 30 on 36g nozzle, 5 V, $50 \mu$ s pulse width, $160 \mu$ m diameter, 24096 fps. Images 1, 7 13, 19, 23, 31, with image 1 just before pulse. The lines used for distance calculation are shown in image 19. 
In general larger droplets were easier to produce since the upper bound of drop size that a nozzle can produce depends on the surface tension holding the drop in place. We focused on creating smaller droplets. Smaller droplets are difficult to separate from the nozzle tip since there is less gravity pulling the droplet against the surface tension forces, resulting in lower-quality droplets. The lower limit of droplet size was observed to occur when the droplet diameter hanging from the end of the nozzle was roughly equivalent to the nozzle diameter. The droplet diameters were measured with the drops hanging from the nozzle in the horizontal direction using ImageJ, and are given within $+/-20 \mu \mathrm{m}$ of the true value. Each drop was measured from the nozzle diameter, and the uncertainty was a result of the resolution of the image.

Qualitative characteristics of each drop were recorded using a point system. These characteristics include: if the drops produced secondary drops (identified visually, drops that were small compared to the primary drop or collected by the primary drop on camera were excluded), if they were significantly off-round exiting the field of view (which changed with camera speed, visually determined), and if they had significant off-axis travel. Run conditions were given quality scores by adding contributions from each of these factors as well as whether or not the primary drop separated from the nozzle. The quality for a given case was scored as follows: oblong drops gained 1 point, off center drops gained 2 points, droplets with secondary drops gained 4 points, and drops that failed to separate gained 8 points. As an example, the droplet in Figure 7 scored a zero as a high-quality drop. If the largest two secondary drops shown were not captured by the primary drop, they would have counted as secondary drops and changed the score to 4. The droplets produced in these experiments are recorded in Figure 8, showing the range of droplet sizes produced. The droplet sizes were selected somewhat randomly, with an emphasis on creating smaller droplets.

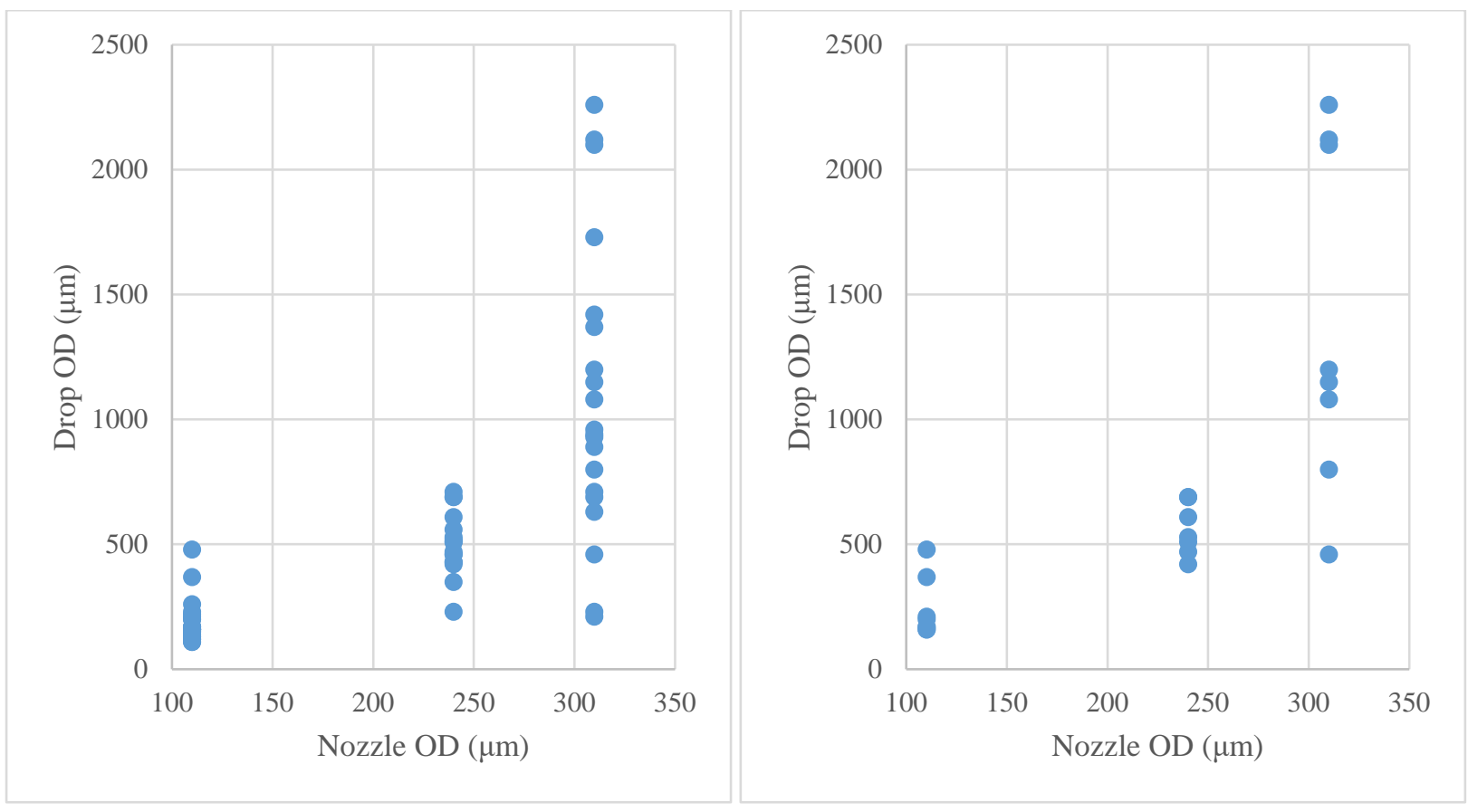

Figure 8 . The range of the droplets produced by the technique. Left: all droplets produced (clean and messy, quality $0-7)$. Right: clean drops only (quality 0 ).

The energy for a given pulse was estimated by assuming that the resistance across the nozzle was 0.5 ohms, and 0 in the wires for the discharge of the capacitor over the pulse duration. The resistance changed as the electrodes wore in, and was not recorded for each run. $0.5 \mathrm{ohms}$ was chosen as a round number representative of observed values measured between several tests. No direct relationship between energy and drop quality was observed, but for the $36 \mathrm{~g}$ nozzle oblong and off-axis motion effects were far more likely for higher energy pulses. The $36 \mathrm{~g}$ nozzle produced the most reliable results since the electrode position was more consistent, only using the finely polished electrodes. An upper limit to the energy put into the needle was a concern since voltages too high would melt the needle. A single $36 \mathrm{~g}$ needle was lost at around $5 \mathrm{~V}$ with a $1 \mathrm{~ms}$ pulse width, so for longer pulses we kept all subsequent testing on the 
$36 \mathrm{~g}$ needle below $3 \mathrm{~V}$. The recorded energy for all nozzles and the recorded electrode distance for the $36 \mathrm{~g}$ nozzle are shown in Figure 9.

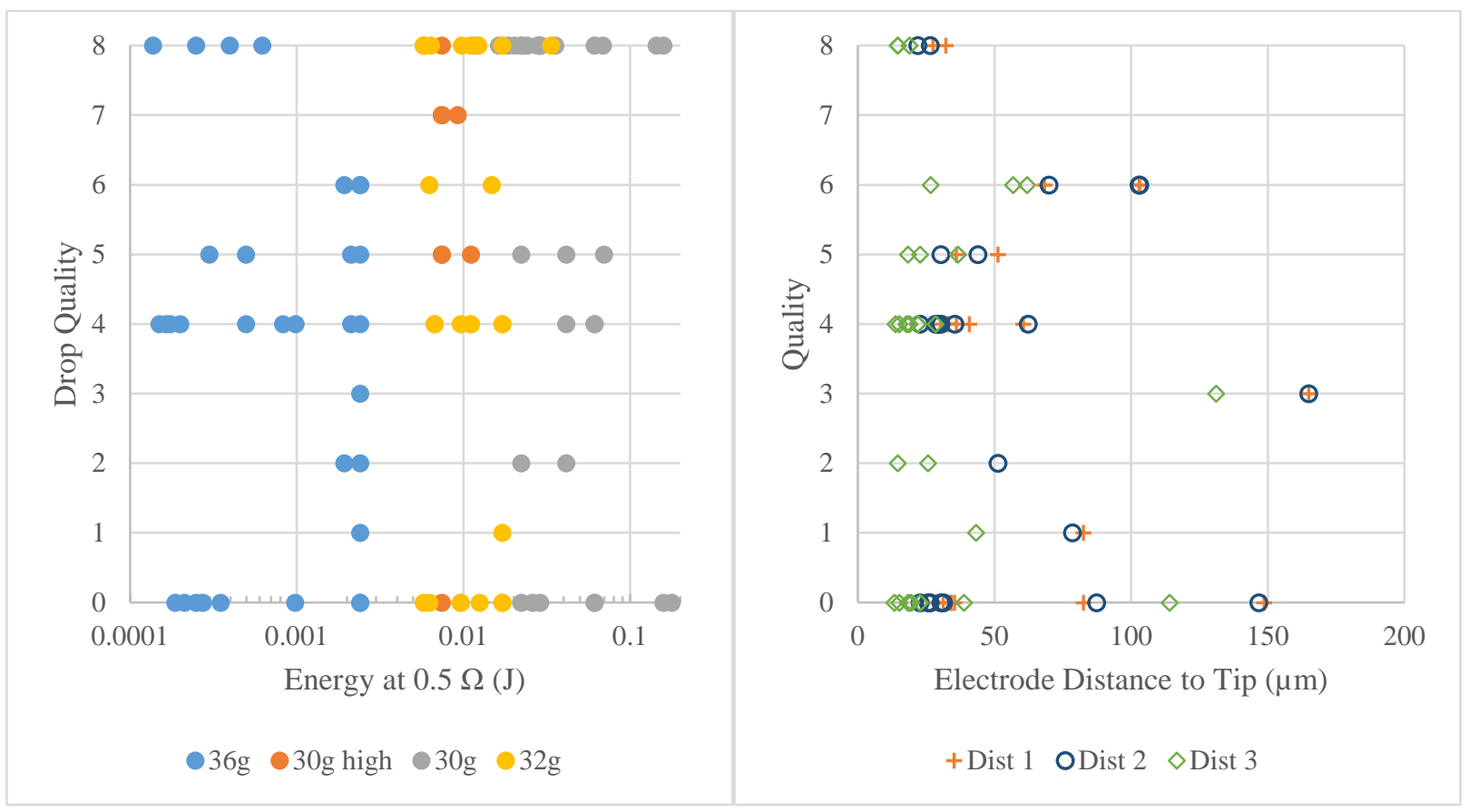

Figure 9. Left: droplet quality vs. estimated pulse energy. Right: drop quality vs. electrode distance for 36g nozzle.

For the 36g needle predictable behavior is observed where, for a particular pulse width there is a minimum voltage that will separate a drop. This cannot be observed in the 30g and 32g needles since electrode position varied much more. Drop size was also observed to have some effect. The electrode height was raised outside of the view of the camera to test producing droplets from within the nozzle by vaporizing water far upstream, similar to a thermal inkjet design. This is depicted in the orange data points in Figure 10. This produced drastically unrepeatable results in the small number of cases tested, and was abandoned. 


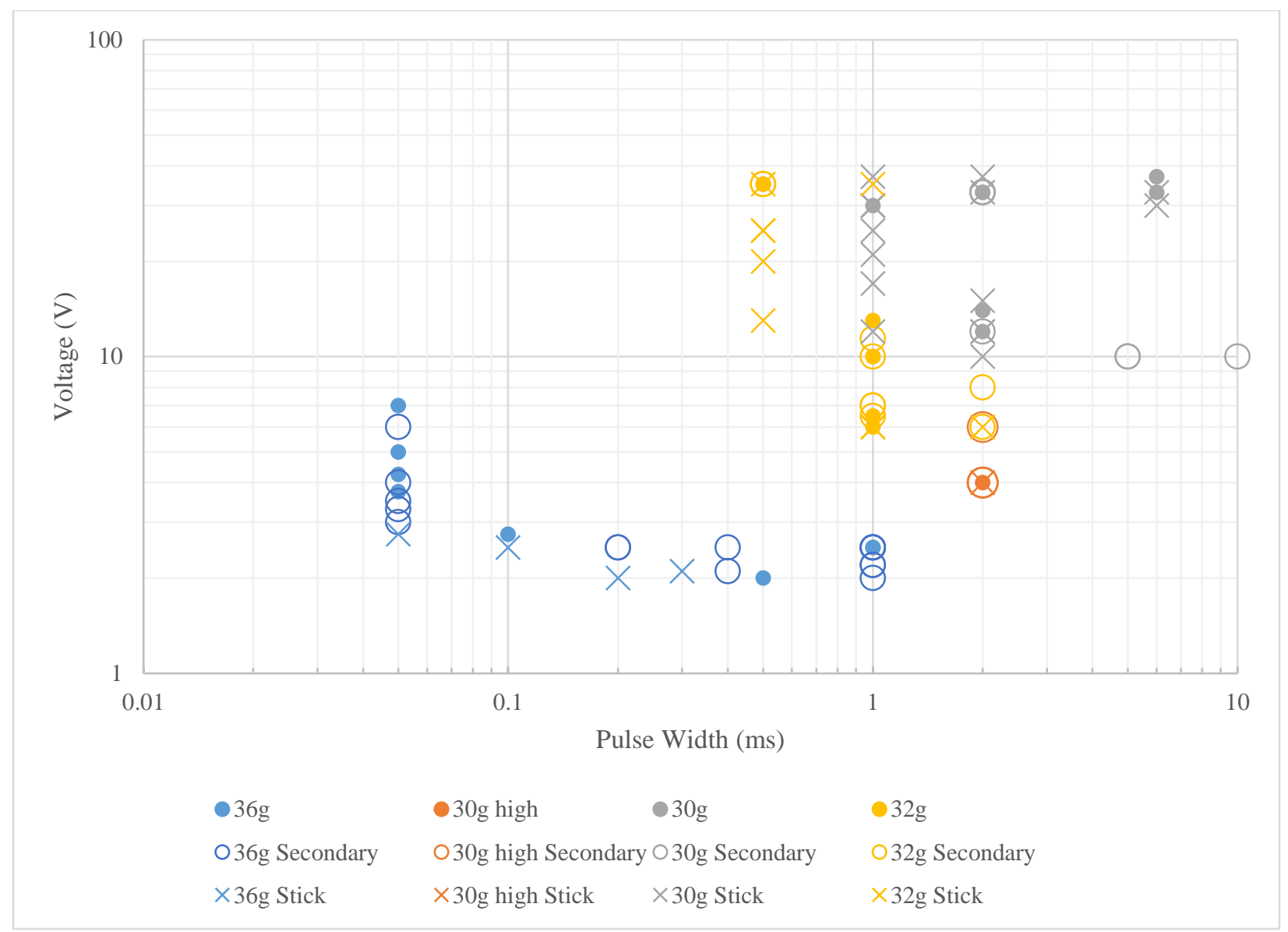

Figure 10. Pulse width, voltage vs. drop produced. The top row in legend represents clean drops, the middle row represents drops with secondary drops, and the last row represents stuck droplets.

Some undesirable effects were not captured in the quality value used above. Vapor bubbles inside the drops were a side effect of the generation technique that wasn't considered in the above analysis since when they were observed it was often on the edge of what could be detected in post-acquisition analysis. Droplets ejecting through the primary drop were counted as secondary drops. Bubbles could be seen coming from the tube into the droplets as they were being ejected. It is unlikely that bubbles existed in the syringe since they were never observed prior to pulsing the nozzle. It is speculated that these are from either water vapor or dissolved gasses in the water. Shorter, higher voltage pulses worked better since the vaporization temperature was more quickly released and less heat overall could be used to separate the droplet. Figure 11 shows an extreme case of bubbles inside a large liquid drop from an early test. 


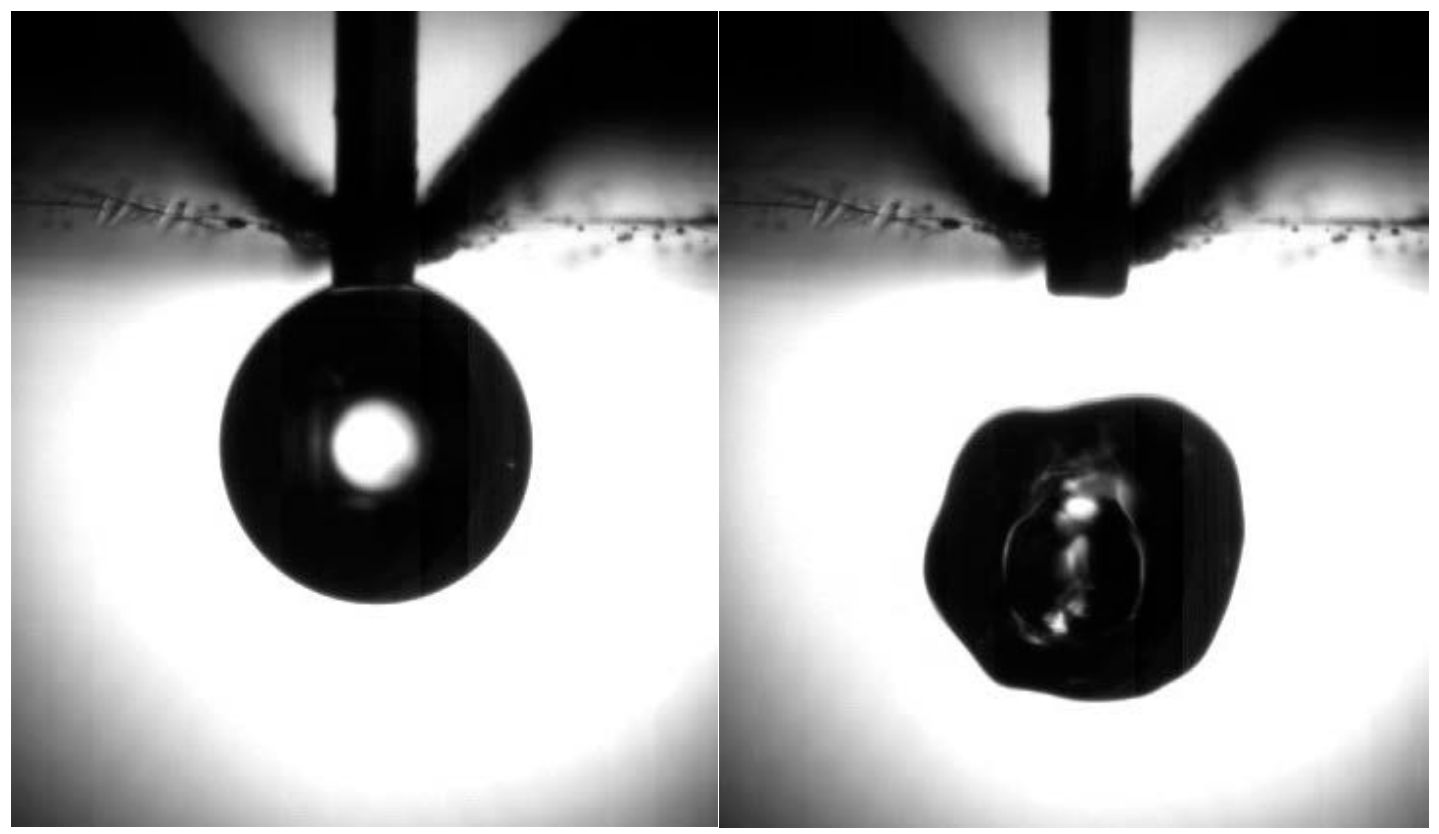

Figure 11. Case 28 on 30g nozzle, 12 V, 2 ms pulse width, $1200 \mu \mathrm{m}$ diameter, 3802 fps. Images 1, 28. Large bubble inserted into drop during release.

In many cases, it was observed that the drop separated from one side of the nozzle last, even with the polished electrodes close to the interface. This indicates that the water around the nozzle rim was not vaporized first, rather water inside the tube upstream from the droplet initiated separation. This was likely the cause of some of the scatter and the off-center motion of the droplets.

In summary, a generator capable of creating single droplets of specified volume was created using only common off-the-shelf components; single droplets were created and separated from a nozzle in a repeatable manner by pulsing electricity across the nozzle. Optimal settings were determined to be short pulse widths at $50 \mu$ around $3 \mathrm{~V}$ using a $36 \mathrm{~g}$ needle, and drops slightly smaller than the needle outer diameter were produced (droplets significantly larger than the needle were trivial to separate). In the range recorded in Figure 9, electrode position had little effect on droplet quality. Undesirable effects were intermittent and difficult to predict for droplets close in size to the nozzle, such as the production of smaller, secondary droplets. Clean droplets were produced as small as $160 \mu \mathrm{m}$ in diameter. The design was able to reliably produce droplets suitable for the study of droplet physics.

\section{Acknowledgements}

The efforts reported in this paper were supported by the NASA Advanced Air Transportation Technology (AATT) Project and the NASA Revolutionary Vertical Lift Technology (RVLT) Project. Any opinions presented in this paper are those of the authors and do not reflect the views of NASA or the United States government.

\section{References}

1. Feo, A., M. Vargas, and A. Sor, Rotating Rig Development for Droplet Deformation/Breakup and Impact Induced by Aerodynamic Surfaces. 2012.

2. Martin, G.D., et al., Droplet generator for a continuous stream ink jet print head. 2003, Google Patents.

3. Meacham, J., et al., Micromachined ultrasonic droplet generator based on a liquid horn structure. Review of Scientific Instruments, 2004. 75(5): p. 1347-1352.

4. $\quad$ Meacham, J., et al., Droplet formation and ejection from a micromachined ultrasonic droplet generator: Visualization and scaling. Physics of Fluids (1994-present), 2005. 17(10): p. 100605.

5. Reis, N., C. Ainsley, and B. Derby, Ink-jet delivery of particle suspensions by piezoelectric droplet ejectors. Journal of Applied Physics, 2005. 97(9): p. 094903.

6. Rezanka, I., Thermal ink jet printhead with droplet volume control. 1991, Google Patents. 
7. Ulmke, H., T. Wriedt, and K. Bauckhage, Piezoelectric droplet generator for the calibration of particlesizing instruments. Chemical Engineering and Technology, 2001. 24(3): p. 265-268.

8. Ulmke, H., et al. The piezoelectric droplet generator; a versatile tool for dispensing applications and calibration of particle sizing instruments. in Proceedings of the 1st International euspen Conference. 1999.

9. $\quad$ Yang, J., et al., A simple piezoelectric droplet generator. Experiments in fluids, 1997. 23(5): p. 445-447.

10. Zoltan, S.I., Pulsed droplet ejecting system. 1972, Google Patents.

11. Christou, K., Droplet generator for an ink jet printer. 1978, Google Patents.

12. Heinzl, J. and W. Schullerus, Droplet generator for generating micro-drops, specifically for an ink-jet printer. 1998, Google Patents.

13. Fan, K.-C., et al., Development of a drop-on-demand droplet generator for one-drop-fill technology. Sensors and Actuators A: Physical, 2008. 147(2): p. 649-655.

14. V Vaught, J.L., et al., Thermal ink jet printer. 1984, Google Patents.

15. Domoto, G.A. and A. Sereny, Thermal ink jet printer with droplet ejection by bubble collapse. 1986, Google Patents.

16. Cheng, S. and S. Chandra, A pneumatic droplet-on-demand generator. Experiments in Fluids, 2003. 34(6): p. 755-762.

17. Goghari, A.A. and S. Chandra, Producing droplets smaller than the nozzle diameter by using a pneumatic drop-on-demand droplet generator. Experiments in Fluids, 2008. 44(1): p. 105-114.

18. Lin, H.B., J. Eversole, and A. Campillo, Vibrating orifice droplet generator for precision optical studies. Review of Scientific Instruments, 1990. 61(3): p. 1018-1023.

19. Abbott, C.E. and T.W. Cannon, A droplet generator with electronic control of size, production rate, and charge. Review of Scientific Instruments, 1972. 43(9): p. 1313-1317.

20. Xu, L., W.W. Zhang, and S.R. Nagel, Drop splashing on a dry smooth surface. Physical review letters, 2005. 94(18): p. 184505.

21. Dong, H., W.W. Carr, and J.F. Morris, An experimental study of drop-on-demand drop formation. Physics of fluids, 2006. 18(7): p. 072102. 